\title{
Quelles retombées éthiques pour le projet Génome français
}

\author{
Hubert Curien
}

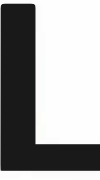

'article que J. Weissenbach a publié dans le numéro d'avril de médecine/sciences se conclut sur des interrogations éthiques d'autant plus intéressantes qu'elles émanent d'un des scientifiques les plus impliqués dans le projet Génome français.

Pour résumer, l'auteur s'interroge sur les capacités d'investigations diagnostiques des applications pratiques du programme et leur impact social. Alors que l'on pourrait se réjouir de voir apparaître de nouveaux outils d'analyse et de détections de maladies invalidantes et parfois mortelles, on peut légitimement s'inquiéter de l'utilisation qui en sera faite.

Les progrès foudroyants de la biologie nous ont apporté des batteries de tests capables de cribler une variété de maladies insoupçonnées il y a tout juste vingt ans. L'irruption de tests originaux ne devrait pas troubler le paysage sauf à considérer que ces nouveaux outils diffèrent des autres sur un point essentiel : leur caractère prédictif. Les méthodes d'investigations traditionnelles révèlent généralement une affection installée ou en cours d'apparition. $\mathrm{Si}$ les retombées du programme Génome français tiennent leurs promesses, les tests dont nous pourrons disposer auront, pour la plupart, un caractère prédictif. En d'autres termes, ils seront capables de prévoir une probabilité d'apparition d'une maladie et ce dès la phase embryonnaire. Cela pose un problème nouveau : faut-il prendre en compte des affections probables mais non avérées. Je considérerai, à titre d'exemple, deux cas de figures à l'opposé l'un de l'autre. Pre$m / s n^{\circ} 5$, vol. 8, mai 92 mier cas, la pratique médicale. A l'évidence, l'utilisation de ces tests s'impose. A la condition, bien entendu, que leurs caractéristiques aient été validées par la pratique: détermination du mode d'utilisation optimale, organisation de conférences de consensus, etc. L'illusion d'un criblage anténatal systématique et généralisé se heurte à trop d'obstacles logiques, médicaux et pratiques pour pouvoir être sérieusement envisagée. Sur ce point, je pense que les philosophes doivent être rassurés, la médecine n'est pas encore capable de dévoiler tous les mystères de l'être humain.

Deuxième cas, l'utilisation de ces types de tests par les compagnies d'assurance. Contrairement au premier cas, ce type d'examen n'a pas pour but d'améliorer l'état sanitaire des individus mais "d'écarter les mauvais risques". Le principe d'un contrat d'assurance est de répartir les risques sur un ensemble homogène d'individus. La méthode statistique employée doit permettre de répartir sur un grand nombre d'individus un risque faible, connu et financièrement insupportable pour la personne concernée. Pour son équilibre financier, la compagnie d'assurance se doit de vérifier que ses assurés ne sont pas atteints, au moment de la souscription, d'une affection pouvant modifier substantiellement la probabilité de survenue de l'événement indésirable. C'est le but de l'électrocardiogramme évoqué par J. Weissenbach. Il met en évidence (par exemple) une maladie coronarienne installée qui a déjà commencé à produire des conséquences pathologiques sur le muscle cardiaque.
Le cas du diagnostic génétique est tout autre. Il ne révèle pas une maladie préexistante, il en prévoit, soit l'apparition probable, soit uniquement la fréquence accrue de survenue future. Comparer les renseignements fournis par ces deux types d'approche reviendrait à réduire l'être humain à son seul programme génétique.

Conscient de ces problèmes, le Gouvernement, à l'issue d'une période de réflexion qui a mêlé les enquêtes parlementaires, les rapports et les débats scientifiques et publics, a déposé sur le bureau des assemblées, trois projets de loi qui devraient donner une réponse légale à certaines de ces interrogations. Il est en effet prévu, dans le projet présenté par le ministre de la Justice, de réserver l'usage des tests génétiques aux seules finalités médicales et de recherche scientifique. Toute autre utilisation devra faire l'objet d'une nouvelle loi qui, le cas échéant, apportera au citoyen des garanties telles qu'une utilisation qui lui porterait préjudice serait proscrite.

Pour ma part, je suis ravi d'un tel débat. Il est sain pour la démocratie et pour l'avancement des sciences. Le vote de ces lois, loin de l'éteindre, devrait lui permettre de se poursuivre au sein et hors de la communauté des chercheurs. Ce n'est qu'en extériorisant pleinement notre mission de service public que les chercheurs rempliront leur vocation qui est avant tout d'être au service de la société

\section{ADRESSE ET TIRÉS À PART}

H. Curien : Ministre de la Recherche et de l'Espace 21, rue Descartes, 75231 Paris Cedex 05, France. 